\title{
Reduction of systems of first-order differential equations via $\Lambda$-symmetries
}

\author{
Giampaolo Cicogna* \\ Dipartimento di Fisica "E.Fermi" dell'Università di Pisa \\ and Istituto Nazionale di Fisica Nucleare, Sez. di Pisa \\ Largo B. Pontecorvo 3, Ed. B-C, I-56127, Pisa, Italy
}

\begin{abstract}
The notion of $\lambda$-symmetries, originally introduced by C. Muriel and J.L. Romero, is extended to the case of systems of first-order ODE's (and of dynamical systems in particular). It is shown that the existence of a symmetry of this type produces a reduction of the differential equations, restricting the presence of the variables involved in the problem. The results are compared with the case of standard (i.e. exact) Lie-point symmetries and are also illustrated by some examples.
\end{abstract}

PACS: 02.20.Sv; 02.30.Hq

Keywords: $\lambda$-symmetries; reduction procedures; dynamical systems

\section{Introduction}

It is a well known property that if an ordinary differential equation (ODE) admits a Lie point-symmetry, then the order of the equation can be lowered by one (see e.g. [1]). The notion of $\lambda$-symmetry has been introduced in 2001 by Muriel and Romero $[2,3]$ with the main purpose of obtaining this reduction even in the absence of standard Lie symmetries. The idea consists in introducing a suitable modification of the prolongation rules of the vector field in such a way that the lowering procedure still works, even if $\lambda$-symmetries are not symmetries in the proper sense, as they do not map in general solutions into solutions.

$\lambda$-Symmetries are related to symmetries of integral exponential type $[1,2,3]$, to hidden and to some classes of potential symmetries (see $[3,4,5]$ and references therein). The meaning of $\lambda$-prolongation has been clarified (together with a possible generalization of the procedure) by means of classical theory

\footnotetext{
*Email: cicogna@df.unipi.it
} 
of characteristics of vector fields [6]. $\lambda$-Symmetries have been extended to partial differential equations $[7,8]$ (and called in that context $\mu$-symmetries), and also interpreted in terms of a deformed Lie derivative in a more geometrical approach [9]. A nontrivial relationship with nonlocal symmetries has been recently pointed out [10]; an interpretation in terms of appropriately defined changes of reference frames has been also proposed [11]. For the implications of $\lambda$ - and $\mu$-symmetries in Noether-type conservation rules see [12, 13].

In the case of first-order ODE's, Lie symmetries cannot lower the order of the equations, but they provide a sort of "reduction" of the complexity of the system, or - more precisely - a reduction of the number of the involved variables (see [1], Theorem 2.66). In this paper, we will restrict precisely to the case of systems of first-order ODE's (with usual regularity and nondegeneracy assumptions: see e.g. [1, 14]), and of dynamical systems (DS) in particular, where the application of $\lambda$-symmetries requires some attention and where they exhibit some relevant peculiarities. We shall prove that some forms of reduction are allowed also in these cases.

An application of $\lambda$-symmetries to systems of ODE's has been already considered in a particular case [15]; in the present paper we want to examine more general situations.

\section{Systems of ODE's}

Let us recall first of all that in the case of a single dependent variable $u=u(t)$ (we shall always denote by $t \in \mathbf{R}$ the independent variable, according to its natural interpretation as the time variable in the case of DS), the first-order $\lambda$-prolongation $X_{\lambda}^{(1)}$ of a vector field $X$

$$
X=\tau(t, u) \frac{\partial}{\partial t}+\varphi(t, u) \frac{\partial}{\partial u}
$$

is defined as

$$
X_{\lambda}^{(1)}=X^{(1)}+\lambda Q \frac{\partial}{\partial \dot{u}}
$$

where $X^{(1)}$ is the standard prolongation $[1,14], \dot{u}=\mathrm{d} u / \mathrm{d} t, \lambda=\lambda(t, u, \dot{u})$ is an arbitrary $C^{\infty}$ function, and $Q=\varphi-\tau \dot{u}$.

Considering systems of equations, and then $q>1$ dependent variables $u_{a}=$ $u_{a}(t)$, the natural extension of definition (2) is

$$
X_{\Lambda}^{(1)}=X^{(1)}+(\Lambda Q)_{a} \frac{\partial}{\partial \dot{u}_{a}}
$$

where the sum over $a=1, \ldots, q$ is understood, with

$$
X=\tau \frac{\partial}{\partial t}+\varphi_{a} \frac{\partial}{\partial u_{a}}, \quad Q_{a}=\varphi_{a}-\tau \dot{u}_{a}
$$

and where now $\Lambda$ is a $q \times q$ matrix of $C^{\infty}$ functions depending on $t, u_{a}, \dot{u}_{a}$. The case $\Lambda=\lambda I$ is the one considered, in the context of DS and also for systems of ODE's of any order, by Muriel and Romero [15]. 
Given a system of $q$ first-order ODE's (we shall assume for simplicity that the number of the equations is the same as the number of dependent variables $\left.u_{a}(t)\right)$

$$
F_{a}\left(t, u_{b}, \dot{u}_{b}\right)=0 \quad a, b=1, \ldots, q
$$

we shall say that this system is $\Lambda$-symmetric under a vector field $X$ if there is a matrix $\Lambda$ such that

$$
\left.X_{\Lambda}^{(1)} F_{a}\right|_{F_{a}=0}=0 .
$$

It is clear from (3) that $\Lambda$ is not uniquely defined: indeed, for any matrix $R$ such that $R Q=Q$ then also $\Lambda^{\prime}=\Lambda R$ satisfies the above condition. This arbitrariness in the definition of $\Lambda$, far from being disturbing, may be useful in practice, as it allows the choice of the more convenient matrix $\Lambda$ in view of the given problem.

We shall say that the system (5) is $\Lambda$-invariant under $X$ if there is a matrix $\Lambda$ such that

$$
X_{\Lambda}^{(1)} F_{a}=0 .
$$

It is not too restrictive to assume that the system of ODE's we are going to consider can be put into a $\Lambda$-invariant form. Indeed, extending to $\Lambda$-symmetries a well known result $[1,6]$, it can be shown that if a system is $\Lambda$-symmetric, then there exists a $q \times q$ matrix $A=A(t, u, \dot{u})$ such that

$$
X_{\Lambda}^{(1)} F_{a}=A_{a b} F_{b} .
$$

It is now enough to prove, applying standard arguments (cf. e.g. $[16,17,18]$ ), the existence of some some $q \times q$ invertible matrix $S$, possibly depending on $t, u, \dot{u}$, such that

$$
X_{\Lambda}^{(1)} S+S A=0
$$

and the (locally) equivalent system $G_{a} \equiv S_{a b} F_{b}=0$ turns out to be $\Lambda$-invariant. We will consider in the following, unless otherwise stated, only $\Lambda$-invariant systems.

The matrix $\Lambda$ plays the role of an additional "unknown" in the determining equations which are deduced from the $\Lambda$-invariance condition

$$
\tau \frac{\partial F_{a}}{\partial t}+\varphi_{b} \frac{\partial F_{a}}{\partial u_{b}}+\left(\varphi_{b}^{(1)}+(\Lambda Q)_{b}\right) \frac{\partial F_{a}}{\partial \dot{u}_{b}}=0
$$

where $\varphi_{b}^{(1)}$ is the coefficient of the standard first-order prolongation, and which clearly strongly depend on the explicit form of the functions $F_{a}$. For instance, in the case where $F_{a}=\dot{u}_{a}-f_{a}(t, u)$, i.e. the case of dynamical systems (see Section 3$)$, these equations are

$$
\{\varphi, f\}_{a}=\frac{\partial}{\partial t}\left(\varphi_{a}-\tau f_{a}\right)-\frac{\partial \tau}{\partial u_{b}} f_{a} f_{b}+\Lambda_{a b} Q_{b},
$$

where $\{\varphi, f\}_{a}=\varphi_{b} \frac{\partial f_{a}}{\partial u_{b}}-f_{b} \frac{\partial \varphi_{a}}{\partial u_{b}}$, which clearly become

$$
\{\varphi, f\}_{a}=(\Lambda \varphi)_{a}
$$


in the case of autonomous systems and time-independent vector fields $X$ with $\tau \equiv 0$ (see [19]).

Let us now introduce "symmetry-adapted" coordinates $w_{a}$ (sometimes also called canonical coordinates) characterized by the property of being invariant under the action of the vector field $X$ :

$$
X w_{a}=\tau \frac{\partial w_{a}}{\partial t}+\varphi_{b} \frac{\partial w_{a}}{\partial u_{b}}=0 ;
$$

they are obtained through the associated characteristic equations

$$
\frac{\mathrm{d} t}{\tau}=\frac{\mathrm{d} u_{a}}{\varphi_{a}} .
$$

In this way we introduce exactly $q$ new variables $w_{a}$; one at least of these, say $w_{q}$, will depend explicitly on $t$, and we will choose this as the new independent variable and call it $\eta$. In particular, if $\tau \equiv 0$, we can choose $\eta=t$. As $(q+1)$-th variable, which will be called $z$, we will take the coordinate "along the action of $X$ ", i.e. such that $X z=1$. Summarizing, the new set of variables is

$$
\eta, w_{\alpha}(\eta), z(\eta), \quad \alpha=1, \ldots, q-1
$$

(among these, $w_{\alpha}$ and $w_{q} \equiv \eta$ are invariant under $X$ ) and clearly do not depend on $\Lambda$.

We have now to write the given vector field $X$ and its first $\Lambda$-prolongation (3) in terms of these coordinates. We get first $X=X^{(1)}=\partial / \partial z$, and we then find that eq. (3) takes the form

$$
X_{\Lambda}^{(1)}=\frac{\partial}{\partial z}+M_{\alpha} \frac{\partial}{\partial w_{\alpha}^{\prime}}+M_{q} \frac{\partial}{\partial z^{\prime}}
$$

where $w_{\alpha}^{\prime}=\mathrm{d} w_{\alpha} / \mathrm{d} \eta, z^{\prime}=\mathrm{d} z / \mathrm{d} \eta$ and (here and in the following the sum will be always understood over the repeated indices $\alpha=1, \ldots, q-1$ and $a=1, \ldots, q$; $D_{t}$ is the total derivative)

$$
\begin{aligned}
& M_{\alpha}=\left(D_{t} \eta\right)^{-2}\left(D_{t} \eta \frac{\partial w_{\alpha}}{\partial u_{a}}-D_{t} w_{\alpha} \frac{\partial \eta}{\partial u_{a}}\right)(\Lambda Q)_{a} \\
& M_{q}=\left(D_{t} \eta\right)^{-2}\left(D_{t} \eta \frac{\partial z}{\partial u_{a}}-D_{t} z \frac{\partial \eta}{\partial u_{a}}\right)(\Lambda Q)_{a} .
\end{aligned}
$$

In particular, if $\tau \equiv 0$, and then with $\eta=t$, we have more simply

$$
\frac{\partial w_{\alpha}^{\prime}}{\partial \dot{u}_{a}}=\frac{\partial w_{\alpha}}{\partial u_{a}} \quad, \quad \frac{\partial z^{\prime}}{\partial \dot{u}_{a}}=\frac{\partial z}{\partial u_{a}}
$$

and

$$
M_{\alpha}=\frac{\partial w_{\alpha}}{\partial u_{a}}(\Lambda Q)_{a} \quad, \quad M_{q}=\frac{\partial z}{\partial u_{a}}(\Lambda Q)_{a}
$$


The above expressions $(13,14)$ can be obtained either by direct calculation expressing by the chain rule the operators $\partial / \partial \dot{u}_{a}$ in terms of $\partial / \partial w_{\alpha}^{\prime}, \partial / \partial z^{\prime}$, or more elegantly - starting from the algebraic relation

$$
\left[X_{\Lambda}^{(1)}, D_{t}\right]=-D_{t}(\tau) D_{t}+(\Lambda Q)_{a} \frac{\partial}{\partial u_{a}}
$$

which can be easily proved and generalizes to $\Lambda$-symmetries other similar known identities $[2,3]$. From this, one directly gets indeed

$$
\begin{aligned}
X_{\Lambda}^{(1)}\left(w_{\alpha}^{\prime}\right) & =X_{\Lambda}^{(1)}\left(\frac{D_{t} w_{\alpha}}{D_{t} \eta}\right)=\frac{X_{\Lambda}^{(1)}\left(D_{t} w_{\alpha}\right)\left(D_{t} \eta\right)-\left(D_{t} w_{\alpha}\right) X_{\Lambda}^{(1)}\left(D_{t} \eta\right)}{\left(D_{t} \eta\right)^{2}}= \\
& =\left(D_{t} \eta\right)^{-2}\left(D_{t} \eta \frac{\partial w_{\alpha}}{\partial u_{a}}-D_{t} w_{\alpha} \frac{\partial \eta}{\partial u_{a}}\right)(\Lambda Q)_{a}=M_{\alpha}
\end{aligned}
$$

thanks to $X_{\Lambda}^{(1)} w_{\alpha}=X w_{\alpha}=0, X_{\Lambda}^{(1)} \eta=X \eta=0$; similarly for $X_{\Lambda}^{(1)}\left(z^{\prime}\right)$.

It can be interesting to point out that eq. (17) puts in clear evidence the difference with respect to exact symmetries: indeed, starting from the $q(X$ invariant) variables $w_{\alpha}, \eta$ one obtains $q-1$ first-order differential quantities $w_{\alpha}^{\prime}$ which are invariant under $X^{(1)}$, but in general not under $X_{\Lambda}^{(1)}$.

In turn, the given system of differential equations will take the form (we will use the $\widetilde{\sim}$ to denote the expressions in the new variables)

$$
\widetilde{F}_{a}\left(\eta, w_{\alpha}, w_{\alpha}^{\prime}, z, z^{\prime}\right)=0
$$

and the condition of its $\Lambda$-invariance under $X$ now becomes

$$
\frac{\partial \widetilde{F}_{a}}{\partial z}+M_{\alpha} \frac{\partial \widetilde{F}_{a}}{\partial w_{\alpha}^{\prime}}+M_{q} \frac{\partial \widetilde{F}_{a}}{\partial z^{\prime}}=0
$$

This allows us to state the following first form of reduction:

Theorem 1. If the system (5) is $\Lambda$-invariant under a vector field $X$, then, once written in the symmetry-adapted coordinates $\eta, w_{\alpha}, w_{\alpha}^{\prime}, z, z^{\prime}$, it turns out to depend on only $2 q$ quantities (instead of $2 q+1$ ): i.e. on the $q$ variables $w_{\alpha}, \eta$ and on other $q$ first-order differential $\Lambda$-invariant quantities $\zeta_{a}=\zeta_{a}\left(\eta, z, w_{\alpha}, w_{\alpha}^{\prime}, z^{\prime}\right)$ which are obtained from the characteristic equations

$$
\mathrm{d} z=\frac{\mathrm{d} w_{\alpha}^{\prime}}{M_{\alpha}}=\frac{\mathrm{d} z^{\prime}}{M_{q}}
$$

coming from condition (19).

Examples 1 and 2 will illustrate this result. 


\section{The case of Dynamical Systems}

Let us now consider the particularly important case of the dynamical systems, i.e. the systems of first-order ODE's which are written "in explicit form":

$$
\dot{u}_{a}=f_{a}(t, u) .
$$

Clearly, once symmetry-adapted coordinates are introduced, the system becomes "automatically" a function of the $2 q$ quantities $w_{\alpha}, \eta, \zeta_{a}$, as granted by Theorem 1.

But it can be preferable or more convenient (e.g. in view of the physical interpretation in terms of "evolution" problem, or also if the explicit expression of the $\zeta_{a}$ is not known ${ }^{1}$ ) to adopt a different point of view, i.e. to preserve the form of the system as an explicit DS, i.e. to rewrite it as follows

$$
\begin{aligned}
w_{\alpha}^{\prime} & =\widetilde{f}_{\alpha}(\eta, w, z) \\
z^{\prime} & =\widetilde{f}_{q}(\eta, w, z) .
\end{aligned}
$$

and to look for the dependence on $z$ of the r.h.s. This point of view will be elucidated by Examples 3 and 4 .

Recalling the expression (12) of the first $\Lambda$-prolongation of $X$, we then easily deduce in this case:

Theorem 2. If a DS is $\Lambda$-invariant under $X$, the dependence on $z$ of the r.h.s. of eq.s (22,23) is given by

$$
\frac{\partial \widetilde{f}_{\alpha}}{\partial z}=M_{\alpha} \quad ; \quad \frac{\partial \widetilde{f}_{q}}{\partial z}=M_{q} .
$$

Then, if for some $\bar{\alpha}$ one has $M_{\bar{\alpha}}=0$, the corresponding $\tilde{f}_{\bar{\alpha}}$ does not depend on $z$. If $M_{\alpha}=0$ for all $\alpha=1, \ldots, q-1$, then only $f_{q}$ depends on $z$ and the system splits into a system for the $q-1$ variables $w_{\alpha}=w_{\alpha}(\eta)$ and the last equation (23) which is an ODE for the variables $z$ and $\eta$.

It is useful to compare the situation covered by Theorems 1 and 2 with the case of exact symmetry: the difference is that in the case of exact symmetry all terms of eq. (18) are independent of $z$; the same is true for all the terms at the r.h.s. of $(22,23)$ : then, in this case, the last equation for $z$ and $\eta$ turns out to be a quadrature, as is well known [1].

Clearly, if $\Lambda=0$ i.e. if $X$ is an exact symmetry, then $M_{\alpha}=M_{q}=0$. Conversely, it can be shown that if $M_{\alpha}=M_{q}=0$ then the symmetry $X$ is exact. This is particularly clear in the case $\tau \equiv 0$ (and then $\eta=t$ ): indeed, in this case the conditions $M_{\alpha}=M_{q}=0$ can be written (see (15)) $\mathbf{J}_{a b}(\Lambda Q)_{b}=0$ where $\mathbf{J}$ is the (invertible!) Jacobian matrix of the transformation from $u_{a}$ to

\footnotetext{
${ }^{1}$ If one is interested to know "a priori" the expressions of the $q$ differential $\Lambda$-invariant quantities $\zeta_{a}=\zeta_{a}\left(\eta, z, w_{\alpha}, w_{\alpha}^{\prime}, z^{\prime}\right)$, one has to express $M_{\alpha}, M_{q}$ in terms of $w_{\alpha}, \eta$ in order to solve $(20)$.
} 
$w_{\alpha}, z$. Then $\Lambda Q=0$, which is the same as $\Lambda=0$ (recall that $\Lambda$ is not uniquely defined).

Notice in particular that the term $(\Lambda Q)_{a}$ appearing in the expressions $(13,14)$, when written in the new coordinates, becomes

$$
(\widetilde{\Lambda} \widetilde{Q})_{a}=\widetilde{\Lambda}_{a q}
$$

indeed $\widetilde{Q} \equiv(0,0, \ldots, 1)$. This shows that only the last column of $\widetilde{\Lambda}$ is relevant.

Finally, let us recall the following result:

Theorem 3. (Muriel-Romero [15]) If $\Lambda=\lambda I$, then $M_{\alpha}=0$ for all $\alpha=$ $1, \ldots, q-1$, and the conclusion of the last part of Theorem 2 holds.

Indeed, from $X w_{\alpha}=X \eta=0, X z=1$ and the definition of $Q$, one easily deduces

$$
Q_{a} \frac{\partial w_{\alpha}}{\partial u_{a}}=-\tau D_{t} w_{\alpha} \quad ; \quad Q_{a} \frac{\partial \eta}{\partial u_{a}}=-\tau D_{t} \eta
$$

and

$$
Q_{a} \frac{\partial z}{\partial u_{a}}=-\tau D_{t} z+1
$$

hence, in the case $(\Lambda Q)_{a}=\lambda Q_{a}$ considered in [15], one gets

$$
M_{\alpha}=0 \quad ; \quad M_{q}=\left(D_{t} \eta\right)^{-2} \lambda .
$$

Notice also that (16) becomes in this case

$$
\left[X_{\lambda}^{(1)}, D_{t}\right]=-D_{t}(\tau) D_{t}+\lambda Q_{a} \frac{\partial}{\partial u_{a}}=-D_{t}(\tau) D_{t}+\lambda X-\lambda \tau D_{t} .
$$

\section{Examples}

Example 1. This is a very simple example, which can provide a clear illustration of Theorem 1. Consider any system $F_{a}\left(t, u_{1}, u_{2}, \dot{u}_{1}, \dot{u}_{2}\right)=0(a=1,2)$ of two first-order ODE's for the variables $u_{1}=u_{1}(t), u_{2}=u_{2}(t)$ and consider the vector field

$$
X=\frac{\partial}{\partial u_{2}} .
$$

It is easily seen that if one chooses

$$
\Lambda=\left(\begin{array}{ll}
0 & 1 \\
0 & 1
\end{array}\right)
$$

then, with our notation, $w_{1}=u_{1}, \eta=t$ and $z=u_{2}$; eq. (13,14) give $M_{1}=$ $M_{2}=1$ and therefore from (20) $\zeta_{1}=\dot{w}_{1}-z=\dot{u}_{1}-u_{2}, \zeta_{2}=\dot{z}-z=\dot{u}_{2}-u_{2}$. Then, $\Lambda$-invariance under $X$ gives that the $F_{a}$ depend only on the quantities $t, \dot{u}_{1}-u_{2}, \dot{u}_{2}-u_{2}$, in agreement with Theorem 1. Extension to more than 2 variables $u_{a}$ is immediate. 
Example 2. Consider a system of ODE's for the two variables $u_{1}=u_{1}(t), u_{2}=$ $u_{2}(t)$ of the form

$$
\begin{aligned}
& h\left(s_{1}, s_{2}\right)\left(\dot{u}_{1}-u_{1} u_{2}\right)+a(t)\left(u_{1}^{2}+u_{2}^{2}\right) u_{1}+b^{2}(t)\left(u_{1}^{2}+u_{2}^{2}\right) u_{2}=0 \\
& h\left(s_{1}, s_{2}\right)\left(\dot{u}_{2}+u_{1}^{2}\right)+a(t)\left(u_{1}^{2}+u_{2}^{2}\right) u_{2}-b^{2}(t)\left(u_{1}^{2}+u_{2}^{2}\right) u_{1}=0
\end{aligned}
$$

where $h$ is a function of $s_{1}=u_{1} \dot{u}_{1}+u_{2} \dot{u}_{2}, s_{2}=u_{1} \dot{u}_{2}-\dot{u}_{1} u_{2}+u_{1}^{3}+u_{1} u_{2}^{2}$ and where $a(t), b(t)$ are arbitrary functions of $t$; it is clearly not symmetric under the rotation operator

$$
X=u_{2} \frac{\partial}{\partial u_{1}}-u_{1} \frac{\partial}{\partial u_{2}}
$$

(unless $h \equiv 0$ ), however it turns out to be $\Lambda$-symmetric (but not $\Lambda$-invariant) under rotations if $\Lambda=\lambda I$ with $\lambda=u_{2}$. Indeed, e.g., one has $X_{\Lambda}^{(1)} h=0$, $X_{\Lambda}^{(1)}\left(\dot{u}_{1}-u_{1} u_{2}\right)=\left(\dot{u}_{2}+u_{1}^{2}\right)$, etc. Introducing symmetry-adapted coordinates, which are $w_{1}=r=\left(u_{1}^{2}+u_{2}^{2}\right)^{1 / 2}, \eta=t, z=\theta$, with obvious notations, the system becomes

$$
\begin{aligned}
& \dot{r} h\left(r \dot{r}, r^{2}(\dot{\theta}+r \cos \theta)\right)+a(t) r^{3}=0 \\
& (\dot{\theta}+r \cos \theta) h\left(r \dot{r}, r^{2}(\dot{\theta}+r \cos \theta)\right)-b^{2}(t) r^{2}=0
\end{aligned}
$$

which turns to be $\Lambda$-invariant under $X=\partial / \partial \theta$ with $\lambda=r \sin \theta$. As expected, thanks to Theorem 1, this system contains only the four quantities $r, t$ and $\zeta_{1}=\dot{r}, \zeta_{2}=\dot{\theta}+r \cos \theta$.

If, e.g., $h=s_{2}$, the system can be also put in the explicit form of a DS:

$$
\dot{r}= \pm(a(t) / b(t)) r \quad \dot{\theta}= \pm b(t)-r \cos \theta
$$

and - as a consequence - according to Theorems 2 and 3 , one (and only one) of the above equations does not contain $z$ (here: $\theta$ ). Then the system can be easily solved.

Example 3. Consider any DS for $u_{a}=u_{a}(t), a=1,2,3$, of the form

$$
\begin{aligned}
& \dot{u}_{1}=h_{1}\left(t, w_{1}, w_{2}\right)+(a-3 b) u_{2} u_{3}+b u_{3}^{3}+h_{2}\left(t, w_{1}, w_{2}\right) u_{3}+h_{3}\left(t, w_{1}, w_{2}\right) u_{3}^{2} \\
& \dot{u}_{2}=h_{2}\left(t, w_{1}, w_{2}\right)+2 u_{3} h_{3}\left(t, w_{1}, w_{2}\right)+a u_{3}^{2} \\
& \dot{u}_{3}=c u_{3}+2 h_{3}\left(t, w_{1}, w_{2}\right)
\end{aligned}
$$

where $a, b, c$ are constants and $h_{a}$ are functions of $t, w_{1}=2 u_{2}-u_{3}^{2}, w_{2}=$ $3 u_{1}-3 u_{2} u_{3}+u_{3}^{3}$. Systems of this form are $\Lambda$-invariant under the vector field

$$
X=u_{2} \frac{\partial}{\partial u_{1}}+u_{3} \frac{\partial}{\partial u_{2}}+\frac{\partial}{\partial u_{3}} \quad \text { with } \quad \Lambda=\left(\begin{array}{ccc}
0 & 0 & (a-3 b) u_{2} \\
0 & 0 & (2 a-c) u_{3} \\
0 & 0 & c
\end{array}\right)
$$

The $X$-invariant quantities are just $w_{1}, w_{2}$, together with $\eta=t$. The coefficients $M_{\alpha}, M_{q}$ (see eq.s $(13,14)$ ), with $z=u_{3}$, are

$$
M_{1}=4(a-c) z \quad, \quad M_{3}=c \quad
$$




$$
M_{2}=3(a-3 b-c) u_{2}-6(a-c) u_{3}^{2}=3(a-3 b-c)\left(w_{1}+z^{2}\right) / 2-6(a-c) z^{2} .
$$

The characteristic equations (20) can then be easily solved to obtain the three first-order differential $\Lambda$-invariant quantities $\zeta_{1}=\dot{w}_{1}-2(a-c) z^{2}, \zeta_{2}=\dot{w}_{2}-$ $(3 / 2)(a-3 b-c) w_{1} z-(3 / 2)(a+b-c) z^{3}, \zeta_{3}=\dot{z}-c z$. Direct calculation shows that this system becomes

$$
\zeta_{a}-g_{a}\left(t, w_{1}, w_{2}\right)=0
$$

where $g_{1}=2 h_{1}, g_{2}=3 h_{1}-3 h_{3} w_{1}, g_{3}=2 h_{3}$, and then contains only the quantities $t, w_{1}, w_{2}, \zeta_{a}$, in agreement with Theorem 1 . If instead one prefers to write the system as an explicit DS, then it is

$$
\begin{aligned}
\dot{w}_{1} & =2(a-c) z^{2}+g_{1}\left(t, w_{1}, w_{2}\right) \\
\dot{w}_{2} & =\frac{3}{2}(a-3 b-c) w_{1} z+\frac{3}{2}(-a-b+c) z^{3}+g_{2}\left(t, w_{1}, w_{2}\right) \\
\dot{z} & =c z+g_{3}\left(t, w_{1}, w_{2}\right) .
\end{aligned}
$$

Now, if $a=c$, the first equation does not contain $z$; if $a=c$ and $b=0$, only the third equation contains $z$, in agreement with Theorem 2. If $a=c=0$, then only the second equation contains $z$ and "plays the role" of the $q$-th equation in our notation. The case $a=b=c=0$ is of course the case of exact symmetry $\Lambda=0$.

Example 4. This is an example with non-autonomous DS and vector field $X$ with $\tau \neq 0$ and therefore $\eta \neq t$. Consider the DS for $u_{a}=u_{a}(t), a=1,2,3$,

$$
\begin{aligned}
& \dot{u}_{1}=t+h_{1}\left(s, w_{1}, w_{2}\right) \exp \left(-\lambda_{1} t\right) \\
& \dot{u}_{2}=1+h_{2}\left(s, w_{1}, w_{2}\right) \exp \left(-\lambda_{2} t\right) \\
& \dot{u}_{3}=u_{2}+h_{2} \exp \left(-\lambda_{2} t\right) \frac{1-\exp \left(u_{2}\left(\lambda_{2}-\lambda_{3}\right)\right)}{\lambda_{3}-\lambda_{2}}+h_{3} \exp \left(-\lambda_{3} u_{2}\right)
\end{aligned}
$$

where $h_{a}$ are nonvanishing functions of $s=u_{2}-t, w_{1}=u_{1}-t^{2} / 2, w_{2}=$ $u_{3}-u_{2}^{2} / 2$. This system is $\Lambda$-invariant under

$$
X=\frac{\partial}{\partial t}+t \frac{\partial}{\partial u_{1}}+\frac{\partial}{\partial u_{2}}+u_{2} \frac{\partial}{\partial u_{3}}
$$

with $\Lambda=\operatorname{diagonal}\left(\lambda_{1}, \lambda_{2}, \lambda_{3}\right)$. We can choose as invariants under $X$ just $w_{1}, w_{2}$ and $\eta=u_{2}-t$, with $z=u_{2}$. It is now more useful to rewrite the system in these coordinates preserving its form of explicit DS, we get then

$$
\begin{aligned}
w_{1}^{\prime} & =\left(h_{1} / h_{2}\right) \exp \left(\left(\lambda_{2}-\lambda_{1}\right)(z-\eta)\right) \\
w_{2}^{\prime} & \left.=\left(\frac{1-\exp \left(\lambda_{2}-\lambda_{3}\right) z}{\lambda_{3}-\lambda_{2}}-z\right)+\left(h_{3} / h_{2}\right) \exp \left(z\left(\lambda_{2}-\lambda_{3}\right)-\lambda_{2} \eta\right)\right) \\
z^{\prime} & =\left(1 / h_{2}\right) \exp \left(\lambda_{2}(z-\eta)\right) .
\end{aligned}
$$

We see that if $\lambda_{1}=\lambda_{2}$, or $\lambda_{2}=\lambda_{3}$ (notice that the case $\lambda_{2}=\lambda_{3}$ is well defined), one of the above equations does not contain $z$ in agreement with Theorem 2; whereas if $\lambda_{1}=\lambda_{2}=\lambda_{3}$ only one equation contains $z$, as stated by Theorem 3; the same happens also if $\lambda_{1}=\lambda_{2}=0$, according to Theorem 2 . 


\section{Concluding remarks}

An interesting property which relates invariance with $\lambda$-symmetries is the following. Consider the case of an autonomous DS $\dot{u}_{a}=f_{a}(u)$ which is $\Lambda$-invariant under a vector field $X$ of the form $X=\varphi_{a}(u) \partial / \partial u_{a}$. If $w=w(u)$ is any invariant under $X$, i.e. $\varphi_{a} \partial w / \partial u_{a}$, then its Lie derivative along $f_{a}$, i.e.

$$
D_{t}^{(f)} w \equiv f_{a} \frac{\partial w}{\partial u_{a}}
$$

satisfies

$$
X\left(D_{t}^{(f)} w\right)=\left(\varphi_{a} \frac{\partial}{\partial u_{a}}\right)\left(f_{b} \frac{\partial}{\partial u_{b}}\right) w=\Lambda_{a b} \varphi_{b} \frac{\partial w}{\partial u_{a}}
$$

hence $X\left(D_{t}^{(f)} w\right)=0$ if $\Lambda=\lambda I$, having used the commutation rule (9) and the invariance property of $w$. On the other hand, eq. (17) gives directly, for $X$ of the above form,

$$
X_{\Lambda}^{(1)}\left(D_{t} w\right)=(\Lambda \varphi)_{a} \frac{\partial w}{\partial u_{a}}
$$

The strong difference is that the latter result is purely algebraic, being a consequence of the relation (16), and expresses a property of the vector field $X$ which holds independently of the presence of any DS (i.e., of any choice of the functions $f_{a}$ ). The former result, instead, states that the time evolution under the dynamics described by the DS $\dot{u}_{a}=f_{a}$ of a quantity $w(u)$ which is invariant under a vector field $X$ preserves this invariance even if $X$ is not a (standard) symmetry of the DS; it is enough to require that $X$ is a $\lambda$-symmetry of the DS.

It can be noticed that the present statement, concerning Lie derivatives, can be suitably extended to the case of several vector fields $X$ for the given DS (see [20], Prop. 2.1).

Several other aspects of $\lambda$-symmetries (and of all their generalizations as well) could be further investigated. Apart from their geometrical interpretation (see the papers quoted in the Introduction), their action on changes of coordinates should be better understood, as well as their general role in finding solutions of differential equations which do not admit standard symmetries: see e.g. [2, $3,5,6]$ and the references therein; compare also, for instance, with [21], for what concerns the problem of finding integrating factors for ODE's and its relationship with symmetry properties.

It can be observed, finally, that any $\operatorname{ODE} \Delta(t, u, \dot{u}, \ddot{u}, \ldots)=0$ of arbitrary order $>1$ can be transformed into a system of first-order ODE's, and therefore our results could be applied also to this case. This is true in principle: the only nearly obvious remark is that one has to consider no longer vector fields of the form $X=\tau(\partial / \partial t)+\varphi(\partial / \partial u)$ involving only the two variables $t$ and $u$, but also extended vector fields $X=\tau(\partial / \partial t)+\varphi_{(0)}(\partial / \partial u)+\varphi_{(1)}(\partial / \partial \dot{u})+\varphi_{(2)}(\partial / \partial \ddot{u})+\ldots$. It is "conceptually" different to look for vector fields of the former or of the latter form; on the other hand, the "concrete effect" of the existence of a symmetry is different in the two contexts (i.e., lowering the order in the case of the ODE's, 
and respectively reducing the presence of the involved variables in the case of first-order systems, as shown). This holds in particular for $\lambda$ - and $\Lambda$-symmetries, where also the prolongation rules of the vector fields are markedly different in the two cases. To emphasize this different role of $\Lambda$-symmetries in the context of first-order systems, it should be perhaps more appropriate to call them $\rho$ symmetries (where $\rho$ stands for "reducing", in contrast with $\lambda$, which could stand for "lowering").

\section{Acknowledgments}

It is a pleasure to thank Giuseppe Gaeta and Diego Catalano Ferraioli for detailed discussions and useful comments. Thanks are also due to one of the referees for his/her accurate and appropriate comments and suggestions, which helped me to improve the presentation of these results.

\section{References}

[1] P.J. Olver, Application of Lie groups to Differential Equations, Springer, Berlin 1986

[2] C. Muriel, J.L. Romero, IMA J. Appl. Math. 66 (2001) 111, and J. Appl. Math. 66 (2001) 477

[3] C. Muriel, J.L. Romero, Theor. Math. Phys. 133 (2002) 1565; and J. Lie theory 13 (2003) 167

[4] B. Abraham-Shrauner, A. Guo, in: Modern Group Analysis, (N.H. Ibragimov et al. ed.s) Dordrecht, Kluwer, 1993, p.1

[5] M.L. Gandarias, E. Medina, C. Muriel, J. Nonlin. Math. Phys. 9 (2002) 47

[6] E. Pucci, G. Saccomandi, J. Phys. A 35 (2002) 6145

[7] G. Gaeta, P. Morando, J. Phys. A 37 (2004) 6955

[8] G. Cicogna, G. Gaeta, P. Morando, J. Phys. A 37 (2004) 9467

[9] P. Morando, J. Phys. A 40 (2007) 11547, and in: Symmetry and Perturbation Theory (SPT2007) (G. Gaeta et al. eds.), World Scientific, Singapore 2007, p. 140

[10] D. Catalano Ferraioli, J. Phys. A 40 (2007) 5479

[11] G. Gaeta, Int. J. Geom. Meth. Mod. Phys. 4 (2007) 807

[12] C. Muriel, J.L. Romero, P.J. Olver, J. Diff. Eqs. 222 (2006) 164

[13] G. Cicogna, G. Gaeta, J. Phys. A 40 (2007) 11899 
[14] G.W. Bluman, S.C. Anco, Symmetry and Integration Methods for Differential Equations, Springer, New York, 2002

[15] C. Muriel, J.L. Romero, in: Proceedings of the I Colloquium on Lie Theory and Applications (I. Bajo and E. Sanmartin eds.), Publicacións Universidade de Vigo, Vigo 2002, p. 143

[16] E.A. Coddington, N. Levinson, Theory of Ordinary Differential Equations, McGraw-Hill, New York 1955

[17] J.F. Cariñena, M.A. Del Olmo, P. Winternitz, Lett. Math. Phys. 29 (1993) 151

[18] G. Cicogna, M. Laino, Rev. Math. Phys. 18 (2006) 1

[19] G. Cicogna, G. Gaeta, Symmetry and Perturbation Theory in Nonlinear Dynamics, Lecture Notes in Physics, LNP monographs Vol. 57, Springer, Berlin 1999

[20] K.P. Hadeler, S. Walcher, J. Nonlin. Sci. 16 (2006) 583

[21] E.S. Cheb-Terrab, A.D. Roche, J. Symb. Comput. 27 (1999) 501 